IOS Press

\title{
Test-retest reproducibility and validity of the back-leg-chest strength measurements
}

\author{
Gill A. Ten Hoor ${ }^{\mathrm{a}, *}$, Kirsty Musch ${ }^{\mathrm{a}}$, Kenneth Meijer ${ }^{\mathrm{b}}$ and Guy Plasqui ${ }^{\mathrm{a}}$ \\ ${ }^{a}$ Department of Human Biology, Nutrition and Translational Research in Metabolism, Maastricht University \\ Medical Center, Maastricht, The Netherlands \\ ${ }^{\mathrm{b}}$ Department of Movement Sciences, Nutrition and Translational Research in Metabolism, Maastricht University \\ Medical Center, Maastricht, The Netherlands
}

\begin{abstract}
.
BACKGROUND: A single measure to characterize overall muscle strength is advantageous because it saves time and costs of evaluation. For this reason, the back-leg-chest (BLC) strength might be an appropriate single measure in characterizing total body strength.

OBJECTIVE: To assess the test-retest reproducibility and smallest real difference (SRD) of the BLC dynamometer in healthy adults and adolescents and to examine whether handgrip, knee-extensor and knee-flexor strength predict BLC strength in healthy adults.

METHODS: Forty-five adults and 58 adolescents were recruited. In a first session back-leg-chest strength, handgrip strength, and additionally, in adults, isometric knee-extensor strength, and knee-flexor strength were measured. In a second session, 2-5 days later, BLC strength was measured again for test-retest reproducibility.

RESULTS: Inter-session correlations of BLC strength were high (all $r$ 's and ICC's $>0.92$ ). Bland-Altman-plots showed high agreement. The SRD and SRD\% were between 14-26, and 19\% and 26\% respectively. Strength variables (handgrip, kneeextensor, and knee-flexor strength) explained $87 \%$ of the variance in BLC strength. A stepwise linear regression showed that dominant knee extensor and flexor strength were the most important significant predictors of BLC strength $\left(r^{2}=0.86\right)$.

CONCLUSIONS: This study demonstrated that the BLC dynamometer has reasonably high test-retest reproducibility and hence may serve in some pertinent situations to be an appropriate tool for clinical, basic and applied research.
\end{abstract}

Keywords: Back-leg-chest dynamometer, knee extensor strength, knee flexor strength, handgrip strength, dynamometry, reproducibility, validity

\section{Introduction}

Skeletal muscle strength is fundamental for physical performance [1] and health [2,3]. Assessment of muscle strength is commonly used in physical examinations to determine possible impairments and physical disabilities, or in experimental settings to examine the effects of interventions. Therefore, it is important to

\footnotetext{
*Corresponding author: Gill A. ten Hoor, Department of Human Biology, Nutrition and Translational Research in Metabolism, Maastricht University Medical Center, P.O. Box 616, 6200 MD Maastricht, The Netherlands. Tel.: +31 43 3881908; E-mail: Gill.tenHoor @MaastrichtUniversity.nl.
}

have simple, valid, and reliable instruments to measure strength.

Muscle strength is typically measured using dynamometry; it is based on having a person exert maximal resistance (force) against a continuously moving (isokinetic) or an immovable (isometric) mechanical lever, using a single muscle or a muscle group. Dynamometry is highly efficient for both clinical and research purposes [4-8], and is often used to validate other strength assessment techniques [9-11]. However a disadvantage of some dynamometers is that they may be too expensive and/or impractical for some clinical settings. The most frequently used dynamometer is the handgrip dynamometer because of its cost ef- 
fectiveness, simplicity and portability [12]. However, the movement patterns performed during the execution of the handgrip test are not comparable to movement patterns of larger muscle groups, or performed in daily life or exercise training programs [4]. To overcome this limitation, the BLC dynamometer might be an effective, simple and portable way to test total body strength.

The BLC dynamometer is a device that measures isometric force produced together by the back, leg and arm muscles. Bethards and colleagues [13] studied test-retest reproducibility of the BLC dynamometer in adults and reported reliabilities of 0.97 or higher, depending on the body position during the test. Moreover, both hand grip strength (HGS) and knee extensor strength (KES) were shown to be related to overall muscle strength [6,14-17] and have therefore often been used to indicate overall limb muscle strength [14, 18,19]. Findings by Bohannon [2] suggest that even though HGS and KES can be used as an indicator for overall limb muscle strength in healthy adults, using these measures solely is not fully recommended in characterizing overall limb muscle strength. A single measure to characterize overall muscle strength is advantageous because it saves time and costs of evaluation. For this reason, BLC dynamometer might be an appropriate alternative single measure in characterizing overall limb muscle strength. Therefore the purpose of this study was twofold: first to examine testretest reproducibility and SRD of the BLC dynamometer in healthy adults and adolescents and second, to examine whether HGS, KES and knee flexor strength (KFS) predict BLCS in healthy adults.

\section{Methods}

\subsection{Subjects}

To investigate test-retest reproducibility of the BLC dynamometer, 45 healthy adults (18-35 years; 23 female) among students and staff of Maastricht University, and 58 healthy adolescents (12-15 years, 30 boys, 28 girls) from a high school in the Netherlands were recruited. Participants with pathologies or disorders compromising the ability to perform maximal strength exercises were excluded. Before participation, informed consent was obtained from all volunteers (and for the adolescents also from the parents). This study was approved by the Research Ethics Board of Faculty of Psychology and Neuroscience, Maastricht
University (ECP-05-09-2012-A1), and conforms with The Code of Ethics of the World Medical Association (Declaration of Helsinki), printed in the British Medical Journal (18 July 1964).

\subsection{Measurements and procedures}

The measurements in the adult population took place in the movement-lab of Maastricht University, whereas the measurements with adolescents took place at the sports-ground of Dutch high school. For this reason, KES and KFS, measured by the Biodex (Biodex medical systems, Shirley, NY, USA), was only examined in adults. All test procedures were instructed by the same investigator.

All adults did a 5 minute warming up on a cycle ergometer, at 60 rotations per minute and a self-selected resistance. The adolescents warmed up by jogging on a self-selected speed. Subsequently, the test sequence started with BLC strength testing, to ensure that the BLC strength test results would not be affected by the Biodex and handgrip measurements. Values of the BLC strength, HGS, and in adults also KES and KFS were obtained on the same testing day (session 1) with three minutes of rest between the measures. In order to determine the test-retest reproducibility of the BLC strength, the test procedure of the BLC dynamometer was repeated for each participant two to five days after the first test occasion, approximately at the same time of day (session 2). Individuals were asked to abstain from intense physical activity in the two days prior to testing, and between the two test sessions, which were 2-5 days apart.

\subsubsection{Height and weight}

Height (in $\mathrm{cm}$ ) and weight (in $\mathrm{kg}$ ) was measured using a SECA 761 analogue scale and a SECA 213 portable stadiometer (SECA Ltd. Medical Scales and measuring systems) with participants wearing light clothing without shoes.

\subsubsection{Back-leg-chest strength}

A calibrated BLC dynamometer (Baseline, New York, USA; see Fig. 1) measures isometric muscle strength, recorded in kilograms $(\mathrm{kg})$ and pounds (lb) of force. When an external force is applied to a handle, which is attached to an adjustable chain, a steel spring compresses and a pointer moves. The dial ranges from 0 to $300 \mathrm{~kg}(0$ to $660 \mathrm{lb})$ in $10 \mathrm{~kg}(10 \mathrm{lb})$ increments. For the test, the length of the chain was adjusted to the participants' height by asking the subject to stand 


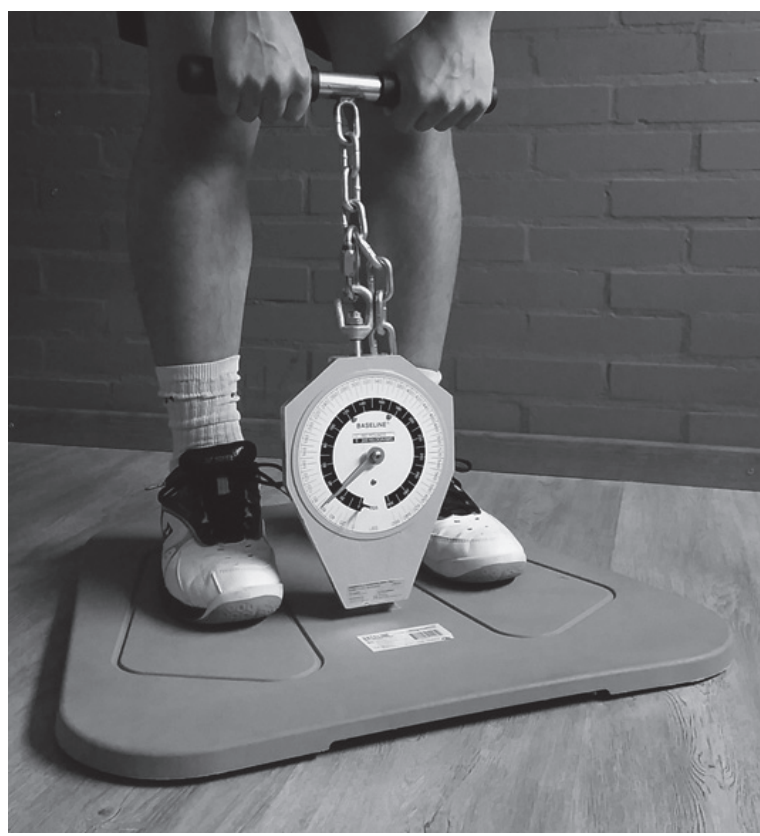

Fig. 1. Back-leg-chest dynamometer.

on the base of the BLC dynamometer with extended knees. Subsequently, the handle was positioned at the height of the intra-articular space of the knee joint. For the test, participants had to stand on the base, with knees and hips flexed slightly while the lower back had to maintain an appropriate lordotic curve. Subjects were asked to lift in a vertical direction by providing continuous isometric contractions of the extensors of the knees, hips, and lower back while holding the handle. Participants were asked to increase the pull in a safe manner gradually and reach the maximal force in three seconds, while keeping this pull for another two seconds. After demonstration and a familiarization trial, three trials were performed, with rest periods of 30 seconds between trials. Maximal strength for the three trials was used for further analysis.

\subsubsection{Handgrip strength}

Dominant and non-dominant HGS was measured using the $\operatorname{Jamar}^{\circledR}$ hydraulic hand dynamometer. The Jamar ${ }^{\circledR}$ handgrip dynamometer is a portable device that measures HGS in kilograms and pounds with increments of $2 \mathrm{~kg}$ (or $2.5 \mathrm{lb}$ ). Isometric HGS was measured according to the American Society of Hand Therapists [20]. In short, the participants sat in a chair without arm rests. The shoulder remained at $0^{\circ}$ flexion, abduction and rotation, the elbow was flexed at $90^{\circ}$ and wrist was positioned between $0^{\circ}$ and $30^{\circ}$ dorsiflexion and between $0^{\circ}$ and $15^{\circ}$ of ulnar deviation. First, a demonstration and a familiarization trial were given for each arm. Then, the participants were instructed to continuously squeeze for 3-5 seconds for three trials, with a 30 second rest period between trials. The maximum value of the three trials was used for further analysis. Testing order (dominant/non-dominant) was balanced. The dominant hand was determined by asking the participants with which hand they write.

\subsubsection{Isometric knee extensor and knee flexor strength}

For measuring isometric KES and KFS, the Biodex System 3 Pro dynamometer was used. Moments were recorded in Newton meters (Nm) with the Biodex Advantage Software for Windows. Participants were in a seated position, whereby the hip and knee joint were flexed at $80^{\circ}$ and $90^{\circ}$ respectively. The knee angle was measured by a goniometer to ensure the appropriate angle of the knee joint. The lateral femoral epicondyle of the knee was lined-up with the axis of the lever arm of the Biodex dynamometer. To prevent compensatory movement, the upper leg and thorax were stabilized with straps. The lever arm was attached just proximal (ca. $3 \mathrm{~cm}$ ) to the ankle joint. Familiarization trials of submaximal effort were performed for both isometric and isokinetic knee flexion and extension. After a short rest period of 30 seconds the actual test was performed, whereby the participants were asked to provide maximal voluntary isometric contractions for 5 seconds at $30^{\circ}, 50^{\circ}, 70^{\circ}, 90^{\circ}$ and $99^{\circ}$ of knee flexion and extension. Resting periods of 30 seconds were applied between each contraction to reduce fatigue and prevent muscular strain. Because the weight of the lower leg and the lever arm of the dynamometer caused passive knee moments, maximal moments had to be corrected for these passive moments. The passive moments were measured before the actual test, by asking the participant to relax the tested leg. Maximal knee flexion and extension strength was determined by fitting a cubic polynomial function to the five joint moment data. The y-top of the polynomial was considered the peak moment and was used for further analysis. The order of Biodex measurements (dominant and non-dominant leg) were balanced, whereby the dominant leg was determined by asking the individuals which leg would be preferred to kick a ball with.

\subsection{Statistical analyses}

IBM SPSS statistics 20 was used for data analysis. Independent samples T-tests were used to identify gen- 
der differences. Test-retest reproducibility of the BLCS was tested using the Intraclass Correlation Coefficient (ICC) in order to determine the correlation and agreement between values. Bland-Altman plots were used to visualize the agreement between the two BLCS-tests and examine the homo- or heteroscedastic nature of the distribution of the differences. The SRD was calculated using the formula: $2.77 \times S D \times \sqrt{ }(1-\mathrm{ICC})$. When correlations between the difference score and mean score $>0.30$, SRD\%'s were calculated. The SRD\% was calculated using the formula SRD $\%=\mathrm{SRD} / M \times 100 \%$. An SRD $\%<30 \%$ was seen as acceptable level of measurement error (see also [21]).

Pearson's correlations were used to identify associations of maximal HGS, isometric knee flexion and extension peak moments, and maximal back-leg-chest strength. A multiple regression model is used to determine whether HGS or KES and KFS predict back-legchest strength. In the regression procedure, the BLCStest of session 1 served as the dependent variable. In the first model, only gender was added as the independent variable. The second model analyzed the following strength variables: HGS of the dominant hand, HGS of the non-dominant hand, KES of the dominant leg, KES of the non-dominant leg, KFS of the dominant leg and KFS of the non-dominant leg. In the third model, gender and the aforementioned strength variables were combined. Stepwise regressions were used to determine the most important predictors.

\section{Results}

A total of 103 participants were examined, 45 adults, and 58 adolescents. Descriptive statistics for the main characteristics of the participants by age and gender are presented in Table 1 . The female and male adults slightly differed by age $(p=0.01)$. Males were significantly taller, heavier, and stronger on all strength tests (all $p^{\prime} s<0.001$ ). In the adolescent population, no differences were found in age ( $p=0.86$ ) or weight ( $p=$ 0.32 ), but boys were signifcantly taller, and stronger on all strength tests (all $p^{\prime} s<0.001$ ).

\subsection{Test retest reproducibility for the $B L C$ dynamometer}

The correlations between the measures of the BLC dynamometer-test in session 1 and session 2 were high for both female $(r=0.92)$ and male $(r=0.93)$ adults, and female $(r=0.83)$ and male $(r=0.94)$ adoles-
Table 1

Study sample characteristics

\begin{tabular}{|c|c|c|c|c|}
\hline & $\begin{array}{l}\text { Total } \\
\text { Mean }(S D)\end{array}$ & $\begin{array}{l}\text { Male } \\
\text { Mean }(S D)\end{array}$ & $\begin{array}{l}\text { Female } \\
\text { Mean }(S D)\end{array}$ & $p$ \\
\hline \multicolumn{5}{|l|}{ Adolescents } \\
\hline $\mathrm{N}$ & 58 & 30 & 28 & \\
\hline Age (year) & $13.9(1.1)$ & $13.8(1.2)$ & $13.9(1.0)$ & 0.84 \\
\hline Height (m) & $168.0(9.2)$ & $171.9(9.4)$ & $163.9(7.0)$ & 0.001 \\
\hline Weight (Kg) & $54.5(9.2)$ & $55.7(9.5)$ & $53.2(8.9)$ & 0.32 \\
\hline BMI $\left(\mathrm{kg} / \mathrm{m}^{2}\right)$ & $19.2(2.1)$ & $18.7(2.0)$ & $19.8(2.2)$ & 0.07 \\
\hline BLC-strength 1 ( $\mathrm{k}$ & $68.1(24.3)$ & $79.2(27.1)$ & $56.3(13.4)$ & $<0.001$ \\
\hline BLC-strength 2 (1 & $73.1(27.1)$ & $84.1(34.4)$ & $60.8(13.7)$ & 0.001 \\
\hline HGS d (Kg) & $34.1(8.5)$ & $37.8(9.5)$ & $30.0(4.8)$ & $<0.001$ \\
\hline HGS nd (Kg) & $32.1(7.6)$ & $36.3(7.8)$ & $27.7(4.2)$ & $<0.001$ \\
\hline \multicolumn{5}{|l|}{ Adults } \\
\hline $\mathrm{N}$ & 45 & 22 & 23 & \\
\hline Age (year) & $25.2(3.6)$ & $26.5(3.9)$ & $23.9(2.8)$ & 0.01 \\
\hline Height (m) & $175.1(9.1)$ & $181.5(6.7)$ & $168.6(6.1)$ & $<0.001$ \\
\hline Weight (Kg) & $72.0(12.6)$ & $81.4(7.9)$ & $63.0(9.1)$ & $<0.001$ \\
\hline BMI $\left(\mathrm{kg} / \mathrm{m}^{2}\right)$ & $23.5(2.8)$ & $24.7(2.7)$ & $22.3(2.4)$ & 0.003 \\
\hline BLC-strength 1 (kg & $93.6(48.9)$ & $132.5(39.0)$ & $56.3(19.0)$ & $<0.001$ \\
\hline BLC-strength $2(\mathrm{~kg}$ & $95.6(43.3)$ & $129.3(33.2)$ & $63.4(22.0)$ & $<0.001$ \\
\hline HGS d (Kg) & $44.2(19.4)$ & $54.7(22.3)$ & $34.0(7.7)$ & $<0.001$ \\
\hline HGS nd (Kg) & $41.7(18.1)$ & $51.8(20.3)$ & $32.0(7.8)$ & $<0.001$ \\
\hline $\operatorname{KES~d~}(\mathrm{Nm})$ & $206.7(73.0)$ & $259.6(63.3)$ & $153.8(32.2)$ & $<0.001$ \\
\hline KES nd (Nm) & $93.2(30.9)$ & $114.2(29.4)$ & $72.3(12.8)$ & $<0.001$ \\
\hline KFS d (Nm) & $197.7(69.9)$ & $253.3(57.1)$ & $146.9(29.0)$ & $<0.001$ \\
\hline KFS nd (Nm) & $92.9(28.8)$ & $112.6(26.7)$ & $75.0(16.2)$ & $<0.001$ \\
\hline
\end{tabular}
Mean $(S D)$ Mean $(S D)$ Mean $(S D) \quad p$

cents. A high degree of reproducibility was found between BLC strength measurements, for male adults an ICC $=0.93$ (95\% CI: 0.90-0.98); for female adults, ICC $=0.92$ (95\% CI: 0.88-0.98); for male adolescents, ICC $=0.83$ (95\% CI: 0.66-0.80); for female adolescents, ICC $=0.83$ (95\% CI: 0.52-0.95). The mean between day variation for BLC strength was $2.67 \pm 12.36$ $\mathrm{Kg}$ in adults, and $1.21 \pm 15.85 \mathrm{Kg}$ in adolescents, with no differences in gender ( $p=0.05$ for adults, and $p=$ 0.64 for adolescents). Bland-Altman plots show testretest reproducibility of the BLC dynamometer in session 1 and session 2 using the error score (see Fig. 2). Correlations between the difference score (Y-axis of Blant-Altman plot) and mean score (X-axis of BlantAltman plot) are $r=-0.36$ for adults, and $r=0.28$ for adolescents. Therefore SRD\%'s are calculated for adolescents, while those for adults should be viewed with reserve.

The SRD\% was calculated using the formula SRD\% $=\mathrm{SRD} / M \times 100 \%$. An $\mathrm{SRD} \%<30 \%$ was seen as acceptable level of measurement error (see also [21, p. 3]).

SRD's and SRD\%'s for male adults (26.2 and $19.9 \%$ ), female adults (15.7 and $26.5 \%$ ) and female adolescents (15.0 and $25.6 \%$ were all within the acceptable level of measurement error. Only the SRD and SRD\% for boys (34.2 and 42.2\%) were not. How- 

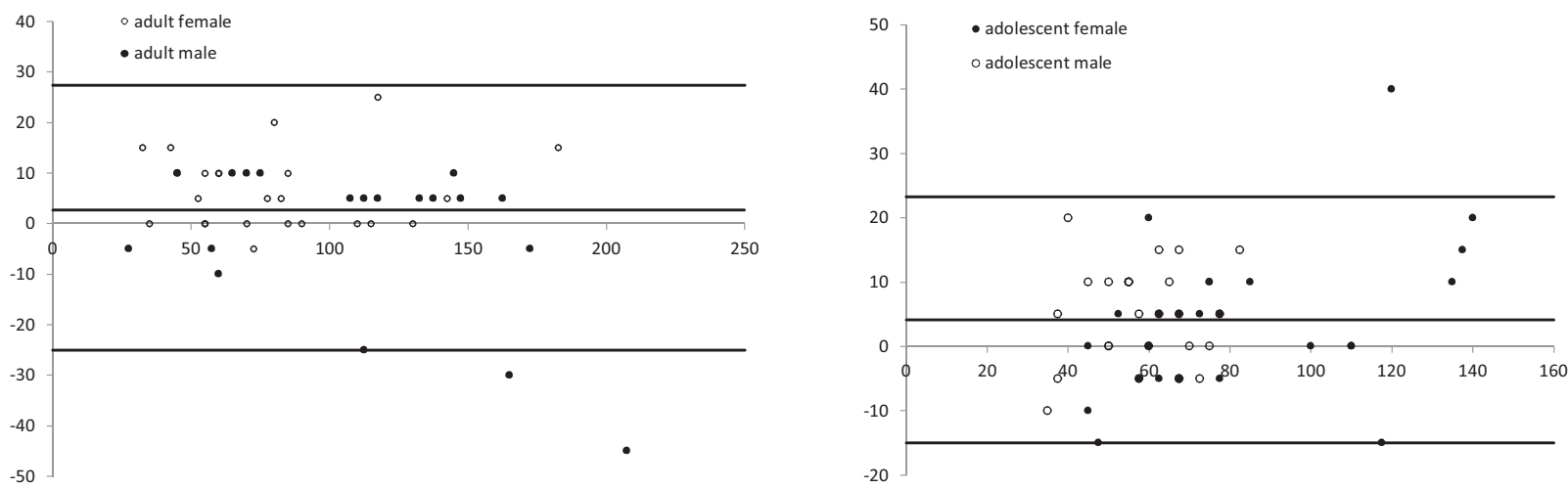

Fig. 2. Blant-Altman plot's indicating the mean difference with limits of agreement between BLC-test 1 and BLC-test 2 in adolescents and adults.

ever, when taking out the one boy that did not fit in the limits of agreement (as clear single outlier; see also Fig. 2), the SRD and SRD\% decreased to an acceptable 17.6 and $22.1 \%$ respectively In very general terms these scores indicate that an increase of at least $20 \%$ will be needed to evidence an improvement in BLC; for a healthy and strong subject this would constitute a major undertaking. On the other hand, it might be a more realistic cutoff in some clinical situations where this type of strength is low and therefore exceeding the $20 \%$ should be a reasonable objective.

\subsection{Strength measure correlates}

In adults (males and females separated), significant correlations were found between the BLC strength and HGS in males, but not in females. All KES and KFS were significantly related to BLC strength. No significant correlations between HGS and KES or KFS were found (all $p$ 's > 0.07; data not shown). In adolescents, BLC strength was significantly correlated with HGS in boys, but not in girls (see Table 2). In Supplemental Digital Content file 2, a full correlation matrix can be found.

Gender predicted $62 \%$ of BLC strength, whereas the strength variables explained a significant proportion of $87 \%$ of the variance. The complete model, with both gender and strength variables, explained variance by $88 \%$ (Table 3). A stepwise linear regression shows that dominant KES and KFS were the most important significant predictors of BLC strength $\left(r^{2}=0.86\right)$.

\section{Discussion}

A single measure to characterize overall muscle strength is advantageous because it saves time and
Table 2

Correlations of handgrip strength, leg extension strength, leg flexion strength, with back-leg-chest strength (session 1)

\begin{tabular}{|c|c|c|c|}
\hline & & \multicolumn{2}{|c|}{ Back-leg-chest strength } \\
\hline \multicolumn{4}{|l|}{ Adolescents } \\
\hline & & Male & Female \\
\hline Dominant hand & Handgrip & $0.71^{* * *}$ & $0.38^{*}$ \\
\hline $\begin{array}{l}\text { Non-dominant hand } \\
\text { Adults }\end{array}$ & Handgrip & $0.78^{* * *}$ & 0.31 \\
\hline Dominant hand & Handgrip & $0.45^{*}$ & -0.04 \\
\hline Non-dominant hand & Handgrip & $0.49^{*}$ & 0.11 \\
\hline \multirow[t]{2}{*}{ Dominant leg } & Extension & $0.65^{* * *}$ & $0.67 * * *$ \\
\hline & Flexion & $0.81 * * *$ & $0.60 * *$ \\
\hline \multirow[t]{2}{*}{ Non-dominant leg } & Extension & $0.56^{* *}$ & $0.71^{* *}$ \\
\hline & Flexion & $0.56^{* *}$ & $0.65^{* * *}$ \\
\hline
\end{tabular}

${ }^{* * *} p<0.001 ;{ }^{* *} p<0.01 ;{ }^{*} p<0.05$.

costs of evaluation. In the past, Bethards and colleagues [13] show a high intra-rater reproducibility of BLC dynamometer measures in adults. Our study replicated the results found by Bethards, but then separately for male and female healthy adults. Additionally, we demonstrated that the BLC dynamometer provides reasonable reproducibility of BLC strength in healthy adolescents. Furthermore, the present study showed that in adult participants, HGS, KES and KFS effectively predict BLC strength measured with the BLC dynamometer. These findings advocate the use of the BLC dynamometer in examining overall limb muscle strength, at least in healthy adults. The results of the present study imply the applicability of the BLC dynamometer. Together with its simplicity and portability, this - up till now seldom used - apparatus might be an appropriate tool in research lab, field research, and clinical settings.

Currently, the usefulness of the BLC dynamometer has been described rarely [22-24]. Coleman and colleagues [22] used the BLC dynamometer in patients with Multiple Myelema, and showed that, fatigue, 
Table 3

Multiple regression analyses predicting back-leg-chest strength from gender (model 1), handgrip strength, leg extension strength, and leg flexion strength (model 2), and a combination (model 3)

\begin{tabular}{llccc}
\hline & & Model 1 & Model 2 & Model 3 \\
\hline \multirow{3}{*}{ Gender } & & Standardized $\beta$ & Standardized $\beta$ & Standardized $\beta$ \\
Dominant hand & Handgrip & -0.79 & - & -0.13 \\
Non-dominant hand & Handgrip & - & -0.16 & -0.18 \\
Dominant leg & Extension & - & 0.27 & 0.27 \\
& Flexion & - & 0.24 & 0.22 \\
Non-dominant leg & Extension & - & 0.50 & 0.43 \\
& Flexion & - & 0.10 & 0.10 \\
Adjusted $\mathbf{R}^{2}$ & & $\mathbf{0 . 6 2}$ & 0.07 & 0.06 \\
\hline
\end{tabular}

pain, sleep, mood and functional performance are interrelated. Sadeghi and colleagues [23] concluded that the BLC dynamometer is not related to heart rate/work hardness. By using the BLC dynamometer, Sener and colleagues [24] found that muscle strength is related to quality of life, depression, and anxiety symptomatology in patients with fibromyalgia.

By using the SRD and SRD\%, a rough relative smallest measurement change that can be interpreted as a real difference was calculated [25]. Although the Blant Altman plots showed some heteroscedasticy, the values found in this study are similar to findings in other studies looking at test-retest reproducibility of isometric strength tests, e.g. in hand function tests [21], and knee extension and flexion tests [26].

\subsection{Other practical applications: Considerations for use}

Several other factors, limitations and strengths have to be considered concerning the use of BLC dynamometer as a measure. First, the apparatus induces execution of static contractions, which are required less often in daily life compared to dynamic contractions. Moreover, rehabilitation and training programs often focus on dynamic training modes. While isometric training is suggested to be more effective than isokinetic training [24-26], this training mode is limited as it increases strength at a specific training angle, but with slight transference to other muscle lengths $[27,28,30]$. Since the BLC dynamometer measures muscle strength in one angle, it provides limited information about muscle function. However, the BLC dynamometer can be used in multiple ways, where different strengths can be measured by instructing different body positions. In this study, specific instructions were given to participants for using the BLCS. Future studies should examine the applicability of the BLC dynamometer for other muscle groups.
Second, some participants experienced the BLC dynamometer test as unpleasant, because of soreness of the lower back during the test that disappeared immediately or within several hours after the test. For this reason, clear instructions about the correct body position, emphasizing the maintenance of an appropriate lordotic curve of the lumbar spine, is essential. Moreover, in order to appease participants, it is important to provide information about possible sensational feelings during and after the test, and explain that these feelings are a normal response to unusual exertion and do not imply tissue damage.

Third, we used two BLC dynamometers in one of our subsequent studies, measuring about 800 healthy adolescents aged 12-15 years in school setting. Both apparatus broke during the experiment (i.e. the pointer stopped moving). Although the apparatus were quickly and easily fixed, our impression is that the inner work (plastic toothed wheels) should be replaced by higher quality materials.

Fourth, our study had a relatively small sample size and examination of the strength predictor variables KFS and KES was studied in adult participants. Therefore, further research is necessary to examine whether these results can be generalized to other age groups.

At last, the difference between the first and second BLC dynamometer measure were within the limit of agreement (except for a few outliers), but sometimes still very large. In each study, the pro's (i.e. portability and testing larger muscle groups outside the lab setting) and con's (variability in measures) need to be considered carefully. For example, for a normal subject to increase the BLC strength reading by $20 \%$ might be a major task. On the other hand, it might serve in some clinical situations where this type of strength is low and therefore exceeding the $20 \%$ should be a reasonable objective. 


\section{Conclusion}

In conclusion, the BLC dynamometer provides reasonably reliable test-retest measurements of BLC strength in healthy adolescents and adults and might therefore be considered an appropriate tool to evaluate changes in muscle strength in research and clinical settings. Additionally, HGS and KES and are shown to be strong predictors of BLC strength in healthy adults, advocating the use of the BLCD in examining overall limb muscle strength.

\section{Supplementary data}

The supplementary files are available to download from http://dx.doi.org/10.3233/IES-160619.

\section{Acknowledgment}

This research was funded by Netherlands Organization for Health Research and Development (ZonMw; project number 525001004).

\section{Conflict of interest}

None of the authors has any conflict of interest. In acknowledgement of concerns regarding lack of disclosure in scientific research, and to aid future metaanalyses, all data, syntax files and extra output, are available as supplemental digital content files (SDC 36) $[31,32]$.

\section{References}

[1] Brill PA, Macera CA, Davis DR, Blair SN, Gordon N. Muscular strength and physical function. Medicine and Science in Sports and Exercise. 2000 Feb; 32(2): 412-6. PubMed PMID: 10694125.

[2] Bohannon RW. Hand-grip dynamometry predicts future outcomes in aging adults. Journal of Geriatric physical Therapy. 2008; 31(1): 3-10. PubMed PMID: 18489802.

[3] Ortega FB, Ruiz JR, Castillo MJ, Sjostrom M. Physical fitness in childhood and adolescence: a powerful marker of health. International Journal of Obesity. 2008 Jan; 32(1): 111. PubMed PMID: 18043605.

[4] Abernethy P, Wilson G, Logan P. Strength and power assessment. Issues, controversies and challenges. Sports Medicine. 1995 Jun; 19(6): 401-17. PubMed PMID: 7676101.
[5] Drouin JM, Valovich-mcLeod TC, Shultz SJ, Gansneder BM, Perrin DH. Reliability and validity of the Biodex system 3 pro isokinetic dynamometer velocity, moent and position measurements. European Journal of Applied Physiology. 2004 Jan; 91(1): 22-9. PubMed PMID: 14508689.

[6] Abbatecola AM, Ferrucci L, Ceda G, et al. Insulin resistance and muscle strength in older persons. The Journals of Gerontology Series A, Biological sciences and medical sciences. 2005 Oct; 60(10): 1278-82. PubMed PMID: 16282559.

[7] Fess EE. The need for reliability and validity in hand assessment instruments. The Journal of Hand Surgery. 1986 Sep; 11(5): 621-3. PubMed PMID: 3760486.

[8] Mathiowetz V, Weber K, Volland G, Kashman N. Reliability and validity of grip and pinch strength evaluations. The Journal of Hand Surgery. 1984 Mar; 9(2): 222-6. PubMed PMID: 6715829.

[9] Dolny DG, Collins MG, Wilson T, Germann ML, Davis HP. Validity of lower extremity strength and power utilizing a new closed chain dynamometer. Medicine and Science in Sports and Exercise. 2001 Jan; 33(1): 171-5. PubMed PMID: 11194105.

[10] Neil SE, Myring A, Peeters MJ, et al. Reliability and validity of the Performance Recorder 1 for measuring isometric knee flexor and extensor strength. Physiotherapy Theory and Practice. 2013 Nov; 29(8): 639-47. PubMed PMID: 23724831.

[11] Taylor JD, Fletcher JP. Correlation between the 8-repetition maximum test and isokinetic dynamometry in the measurement of muscle strength of the knee extensors: A concurrent validity study. Physiotherapy Theory and Practice. 2013 May; 29(4): 335-41. PubMed PMID: 23035768.

[12] Fess EE. A method for checking Jamar dynamometer calibration. Journal of Hand Therapy. 1987; 1(1): 28-32.

[13] Bethards S, Everitt-Smith S, Roberts H, Scarborough G, Tate $\mathrm{S}$, Bandy WD. Intrarater test-retest reliability of an instrument used to measure back and leg strength. Isokinetics and Exercise Science. 1995; 1(5): 31-35.

[14] Bohannon RW. Dynamometer measurements of grip and knee extension strength: are they indicative of overall limb and trunk muscle strength? Perceptual and Motor Skills. 2009 Apr; 108(2): 339-42. PubMed PMID: 19544938.

[15] Bohannon RW. Are hand-grip and knee extension strength reflective of a common construct? Perceptual and Motor Skills. 2012 Apr; 114(2): 514-8. PubMed PMID: 22755456.

[16] Felicio DC, Pereira DS, Assumpcao AM, et al. Poor correlation between handgrip strength and isokinetic performance of knee flexor and extensor muscles in community-dwelling elderly women. Geriatrics \& Gerontology International. 2014 Jan; 14(1): 185-9. PubMed PMID: 23617580.

[17] Rantanen T, Guralnik JM, Foley D, et al. Midlife hand grip strength as a predictor of old age disability. JAMA: The Journal of the American Medical Association. 1999 Feb 10; 281(6): 558-60. PubMed PMID: 10022113.

[18] Bohannon RW. Is it legitimate to characterize muscle strength using a limited number of measures? Journal of Strength and Conditioning Research/National Strength \& Conditioning Association. 2008 Jan; 22(1): 166-73. PubMed PMID: 18296971.

[19] Hosler WW, Morrow Jr JR. Arm and leg strength compared between young women and men after allowing for differences in body size and composition. Ergonomics. 1982 Apr; 25(4): 309-13. PubMed PMID: 7128565.

[20] Fess EE. Grip strength. In: Casanova JS, editor. Clinical assessment recommendations. 2nd ed. Chicago: American Society of Hand Therapists. 1992. p. 41-5. 
[21] Chen, H. M., Chen, C. C., Hsueh, I. P., Huang, S. L., \& Hsieh, C. L. (2009). Test-retest reproducibility and smallest real difference of 5 hand function tests in patients with stroke. Neurorehabilitation and neural repai, p. 3 .

[22] Coleman EA, Goodwin JA, Coon SK, et al. Fatigue, sleep, pain, mood, and performance status in patients with multiple myeloma. Cancer Nursing. 2011 May-Jun; 34(3): 21927. PubMed PMID: 21522061. Pubmed Central PMCID: 3086027.

[23] Sadeghi N, Tolide-ie H, Ghaderi F. The relationship between heart rate as an indicator of work hardness and results of dynamometry. Work. 2012; 41 Suppl 1: 5677-80. PubMed PMID: 22317650.

[24] Sener U, Ucok K, Ulasli AM, et al. Evaluation of healthrelated physical fitness parameters and association analysis with depression, anxiety, and quality of life in patients with fibromyalgia. International Journal of Rheumatic Diseases. 2013 Nov 30. PubMed PMID: 24289723.

[25] Beckerman, H., Roebroeck, M. E., Lankhorst, G. J., Becher, J. G., Bezemer, P. D., \& Verbeek, A. L. M. (2001). Smallest real difference, a link between reproducibility and responsiveness. Quality of Life Research, 10(7), 571-578.

[26] Adsuar, J. C., Olivares, P. R., del Pozo-Cruz, B., Parraca, J. A., \& Gusi, N. (2011). Test-retest reliability of isometric and isokinetic knee extension and flexion in patients with fibromyalgia: Evaluation of the smallest real difference. Archives of physical medicine and rehabilitation, 92(10), 1646-1651.
[27] Thepaut-Mathieu C, Van Hoecke J, Maton B. Myoelectrical and mechanical changes linked to length specificity during isometric training. Journal of Applied Physiology. 1988 Apr; 64(4): 1500-5. PubMed PMID: 3378984.

[28] Weir JP, Housh TJ, Weir LL, Johnson GO. Effects of unilateral isometric strength training on joint angle specificity and cross-training. European Journal of Applied Physiology and Occupational Physiology. 1995; 70(4): 337-43. PubMed PMID: 7649145.

[29] Young K, McDonagh MJ, Davies CT. The effects of two forms of isometric training on the mechanical properties of the triceps surae in man. Pflugers Archiv: European Journal of Physiology. 1985 Dec; 405(4): 384-8. PubMed PMID: 4080515.

[30] Lindh M. Increase of muscle strength from isometric quadriceps exercises at different knee angles. Scandinavian Journal of Rehabilitation Medicine. 1979; 11(1): 33-6. PubMed PMID: 419396.

[31] Peters G-JY, Abraham CS, Crutzen R. Full disclosure: doing behavioural science necessitates sharing. European Health Psychologist. 2012; 14: 77-84.

[32] Simmons JP, Nelson LD, Simonsohn U. False-positive psychology: Undisclosed flexibility in data collection and analysis allows presenting anything as significant. Psychological Science. 2011; 22: 1359-66. 